\title{
A text-mining approach to assess impacts and benefits of Nature-Based Solutions
}

Leydy Alejandra Castellanos Díaz (1), Pierre Antoine Versini (1), loulia Tchiguirinskaia (1) and Olivier Bonin (2)

(1) HM\&Co, Ecole des Ponts ParisTech, UPE, Champs-sur-Marne, France (leydy.castellanos@enpc.fr),

(2) LVMT, Ecole des Ponts ParisTech, UPEM, IFSTTAR, Champs-sur-Marne, France
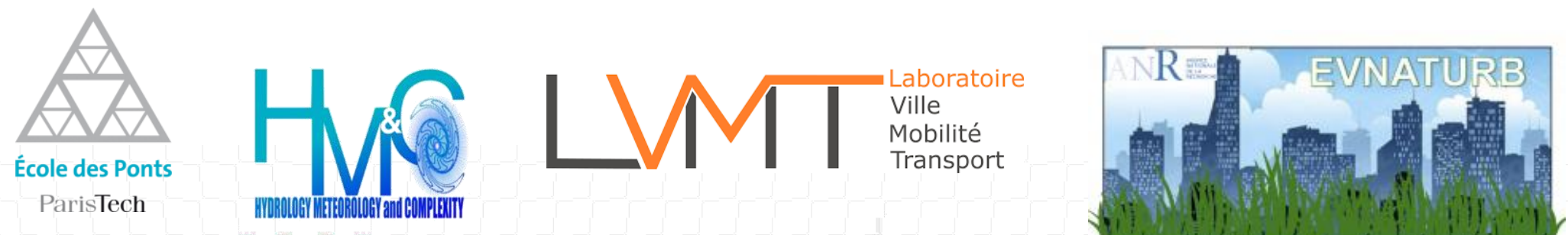


\section{Urban Sustainability Challenges}

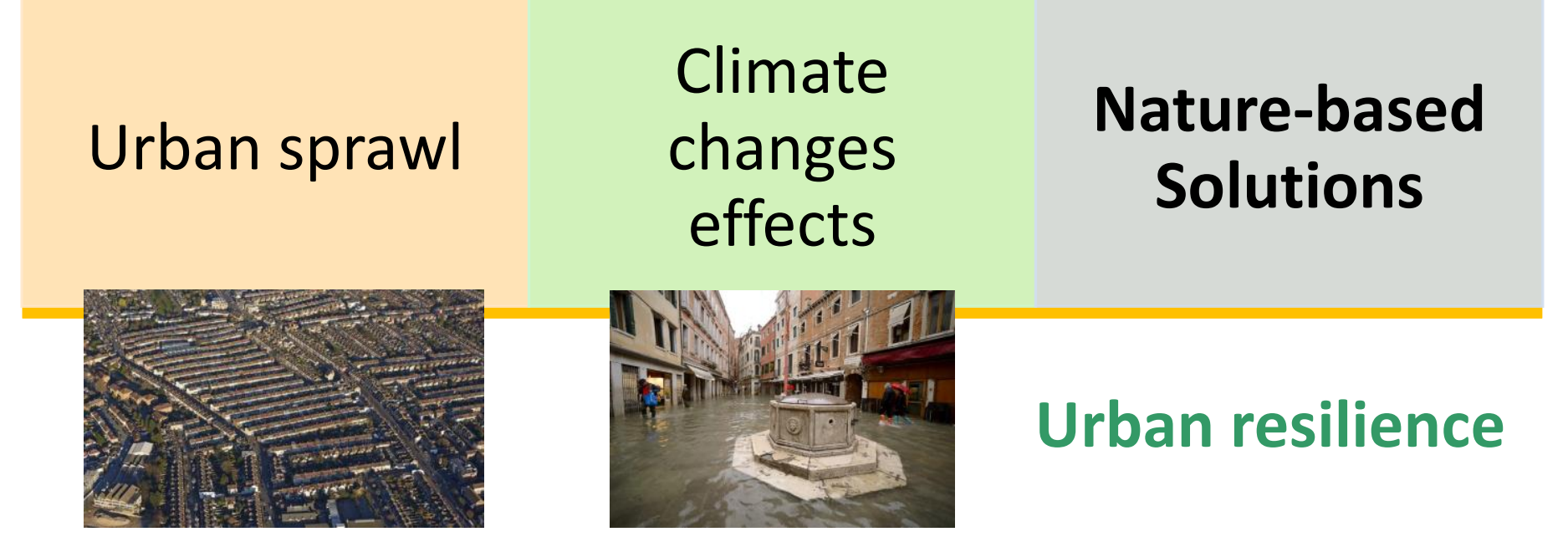

NbS "living solutions inspired and continuously supported by nature, which are designed to address various societal challenges in a resource-efficient and adaptable manner and to provide simultaneously economic, social, and environmental benefits" EC, 2015

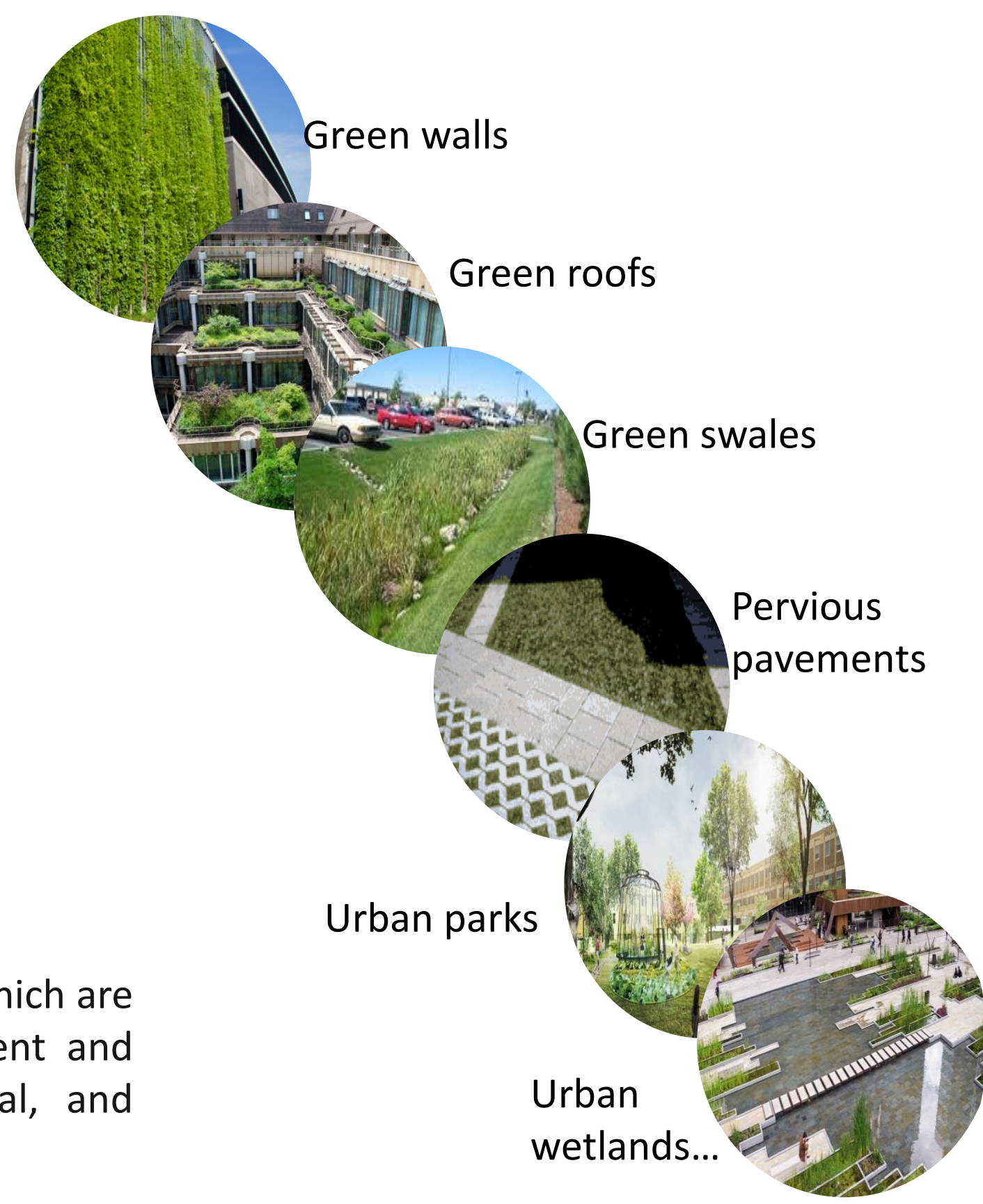




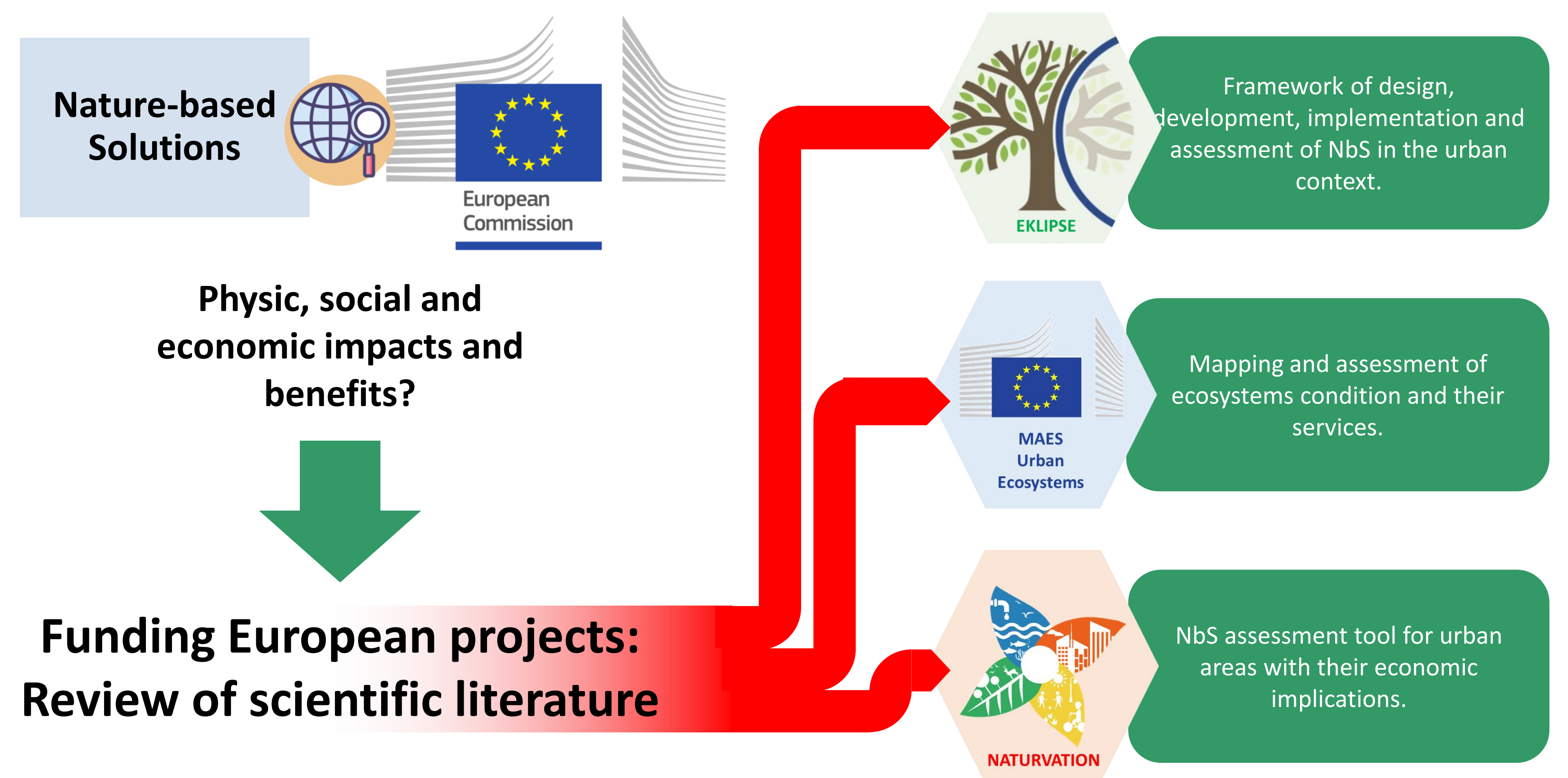
(c) (1) 


\section{Literature review on}

\section{Nature-based solutions}

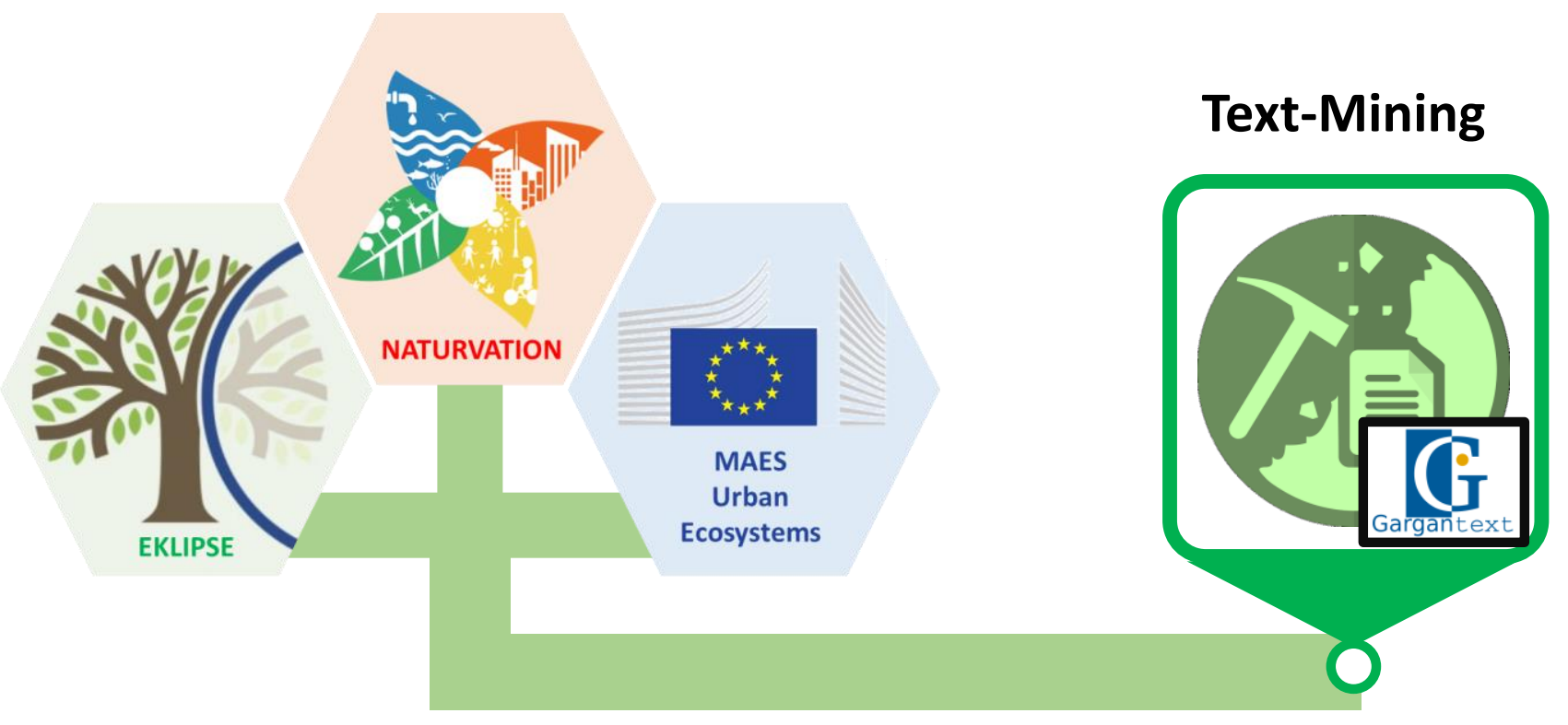

Objective analysis and comparison of documents

\section{Traditional analysis}

Metadata on scientific publications (abstracts, keywords, title, date of publication and author).
Converting text data into a meaningful structured analysis, allowing to recognize concepts as well as to identify stakeholders and their position.

\section{This analysis}

Each report divided in smaller units. 


\section{Literature review on}

Nature-based solutions

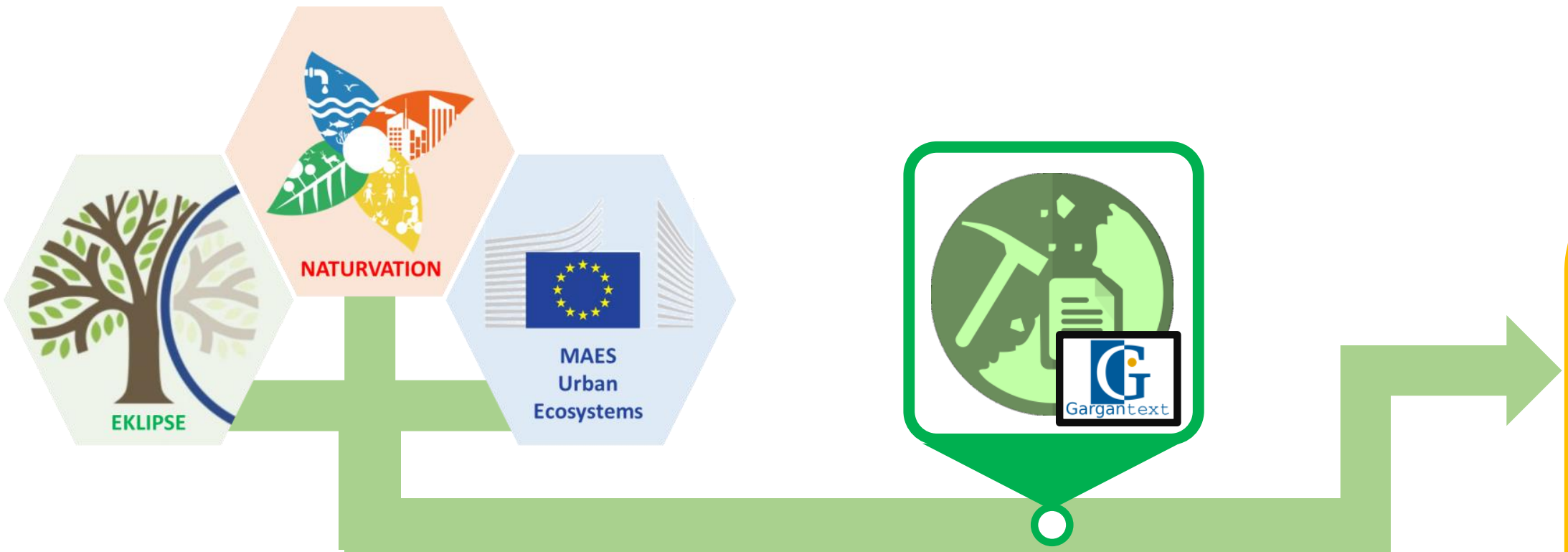

Text-Mining

Occurrence of terms in the report

\section{Statistical analysis:}

- Degree of nodes: number of edges connected to a node

- Edge weight: strength of link between nodes

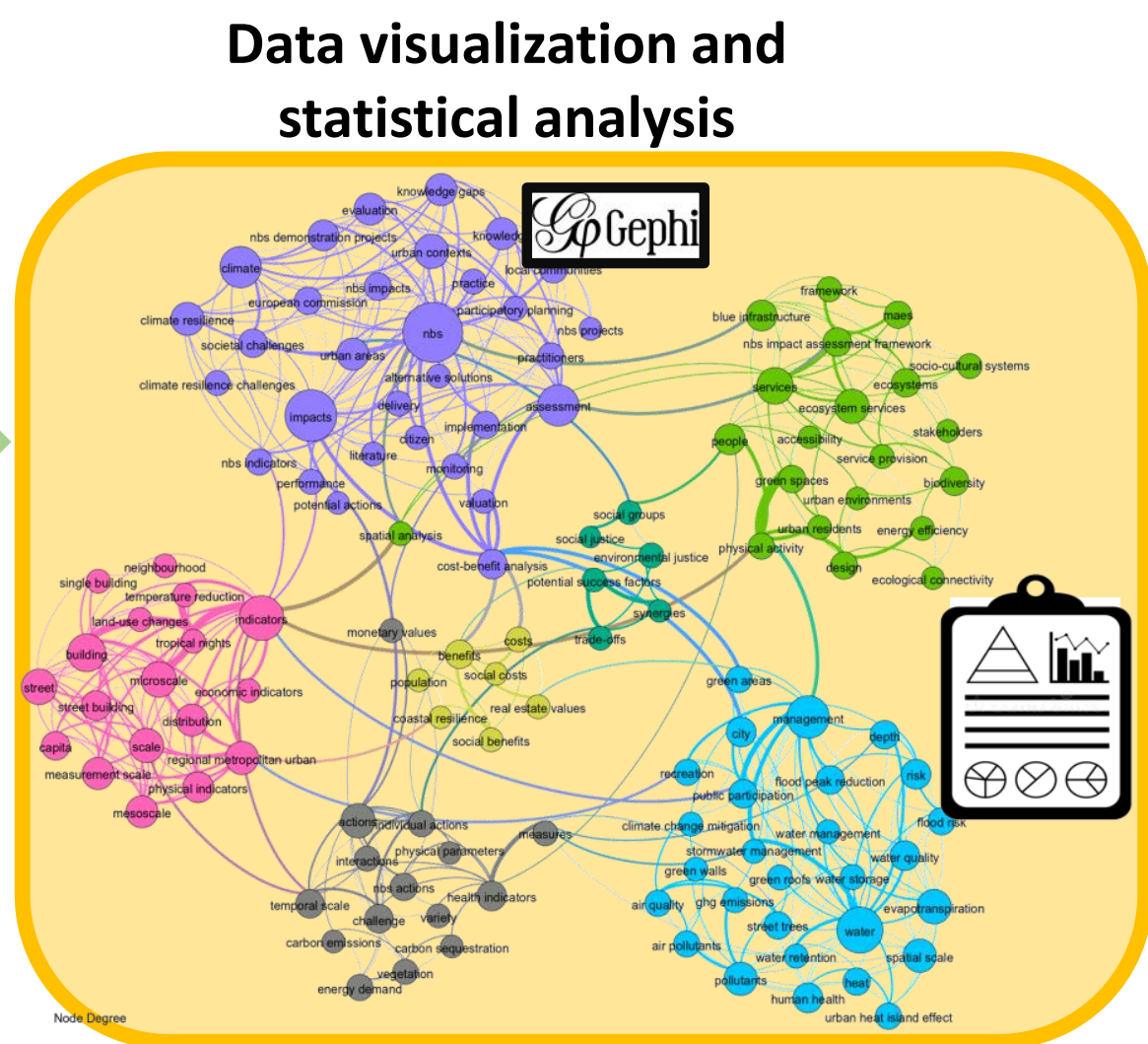

Network:

- Node: key terms

- Edge: connections between nodes

- Clusters: communities of nodes 


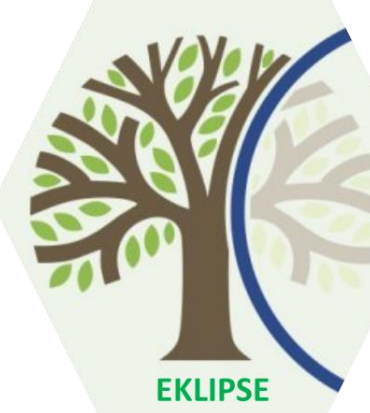

(1) NBS planning, governance

2 NBS to tackle urban

challenges

(3) NBS indicators

(4) Ecosystem services provided by NBS

(5) Action, temporal scale

6) Social and economic benefit of NBS

(7) Social opportunities

\section{Number of nodes}

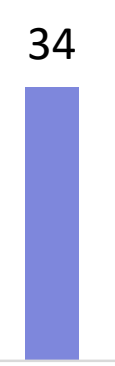

$30 \quad 30$

18

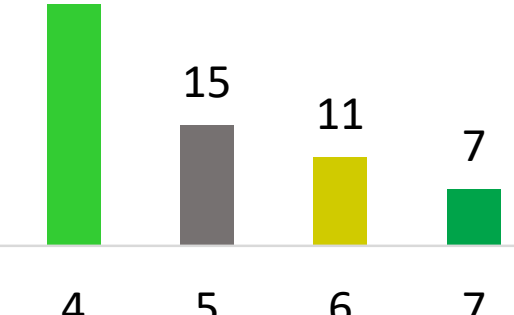

1

Curopean commission impacts climafe resitience european conmission participatory planning Tsocietal chalienges nbs nbs projects climate resilience challenges
$+9$

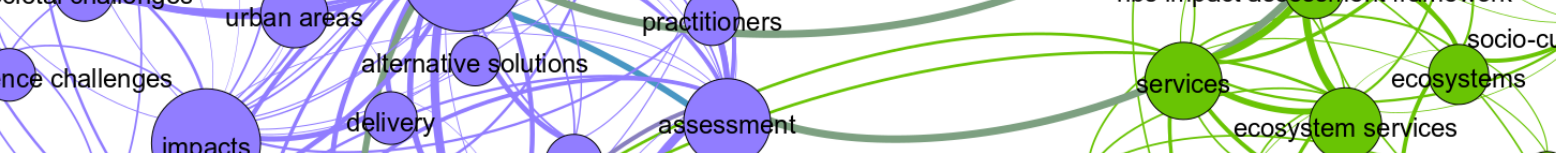

nbs indicators literatyre itizen implemen
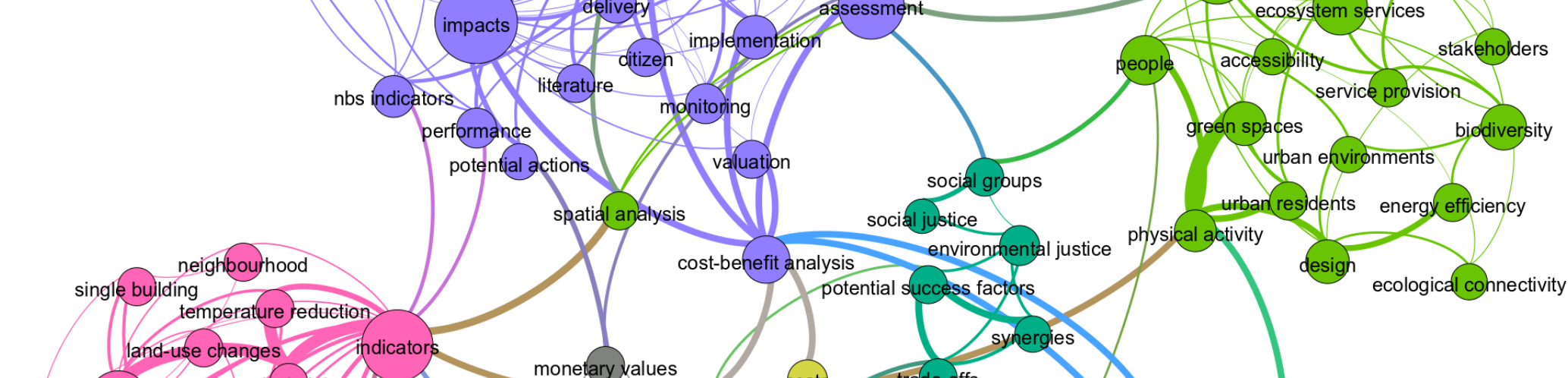

Accumulated Degree

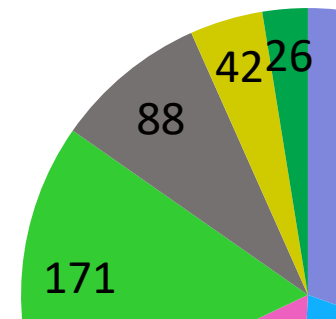

311

171

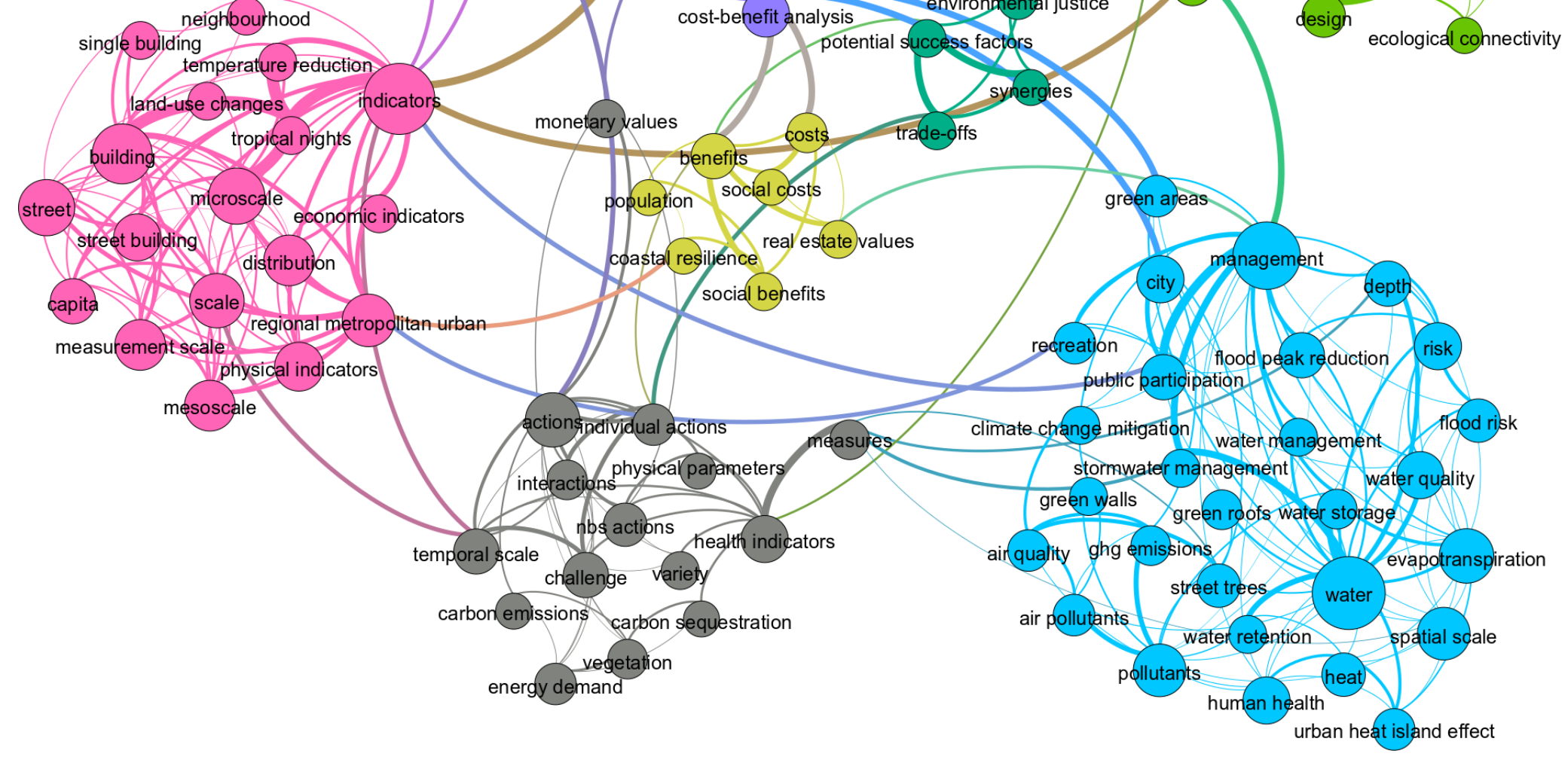


(1) Urban policies and NBS

(2) Ecosystem services provided by NBS

(3) Urban land use

(4) Urban planning

(5) Ecosystem services assessment

(6) Tools to asses

ecosystem services

\section{Number of nodes}

47

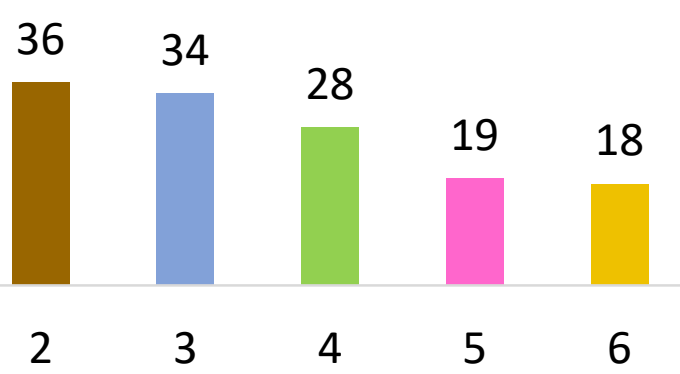

\section{Accumulated Degree}
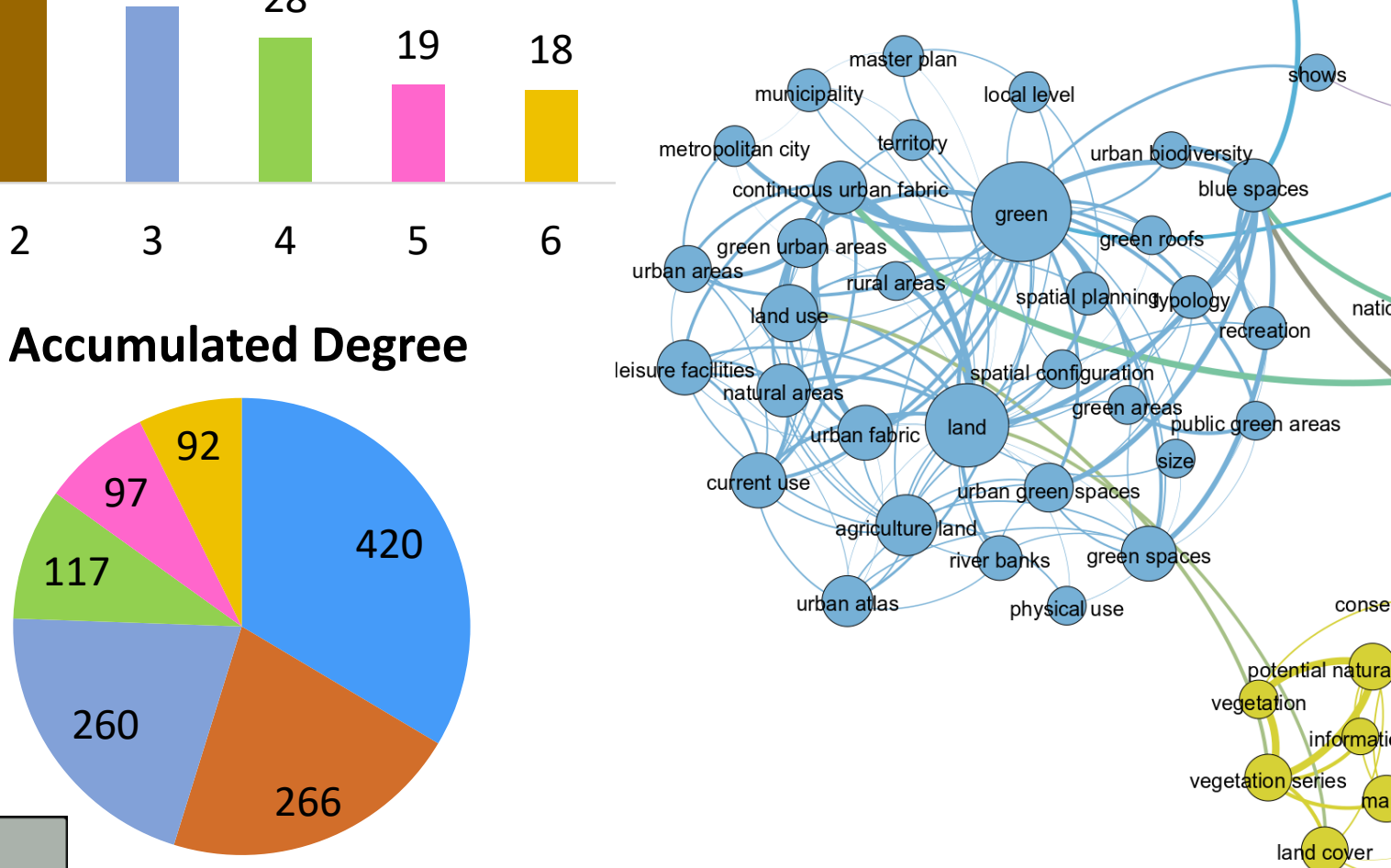

urban areas ans rural areas spatial planningy pology

city leyel urban spraw urban parks
implementation city level urpan sprawl
air polltion municipal level
carbon storage measure

leisure facilities
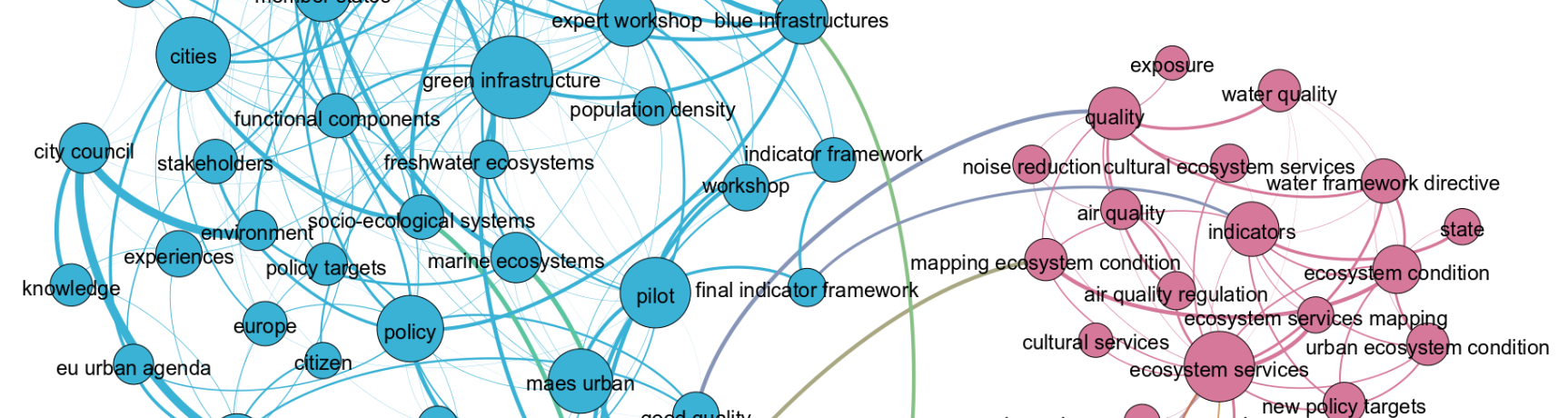

key urban ecosystem services nellicy targets

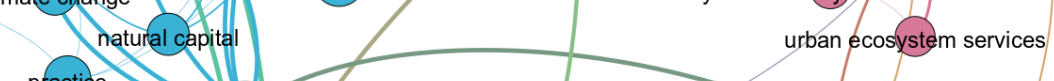

practice urban ecosystem services

practice locan policynaking

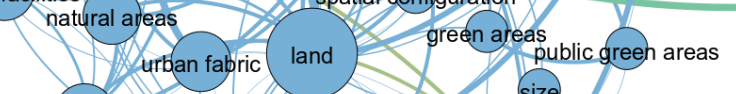

size public gre
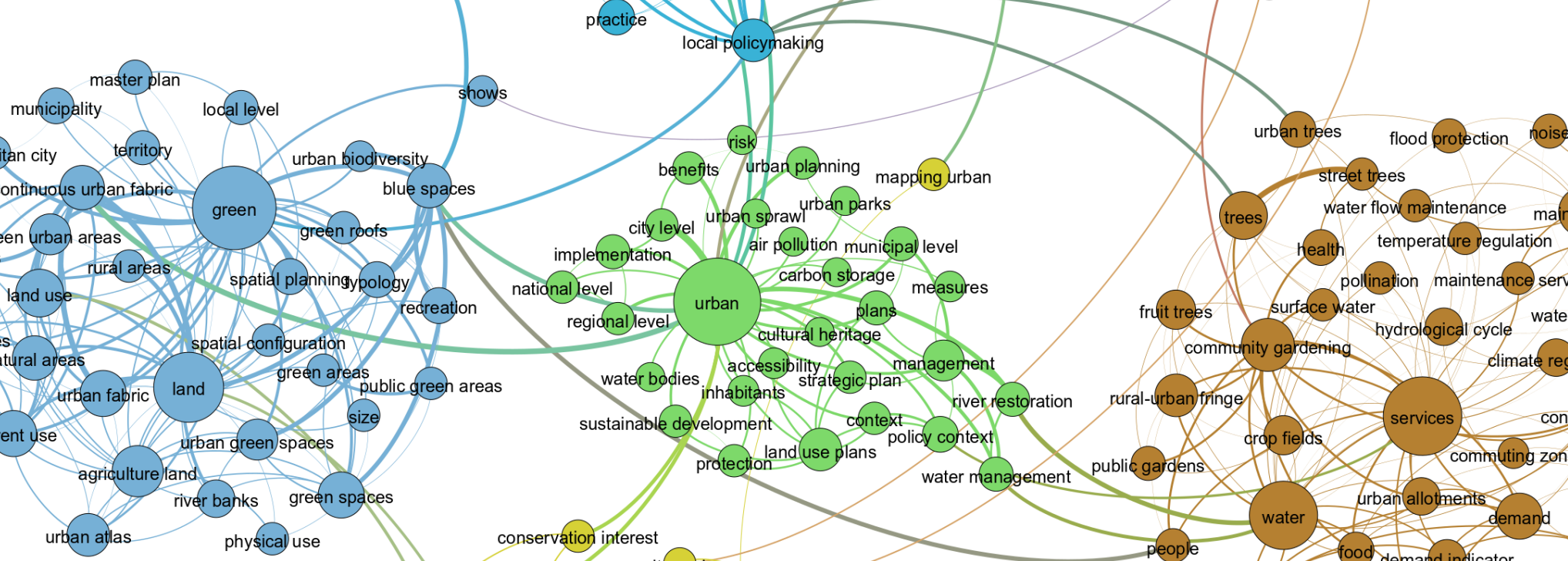

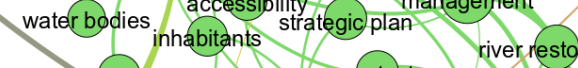
sustainable development context river restoricy context

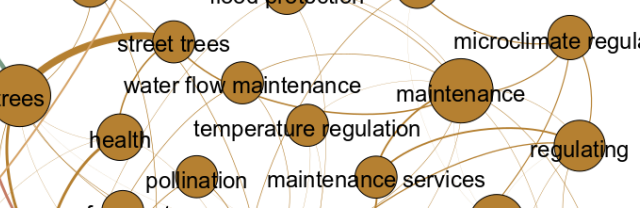

fruit troes sulface water community gardening hydrolegical cycle climate regulation

urban atlas physical use
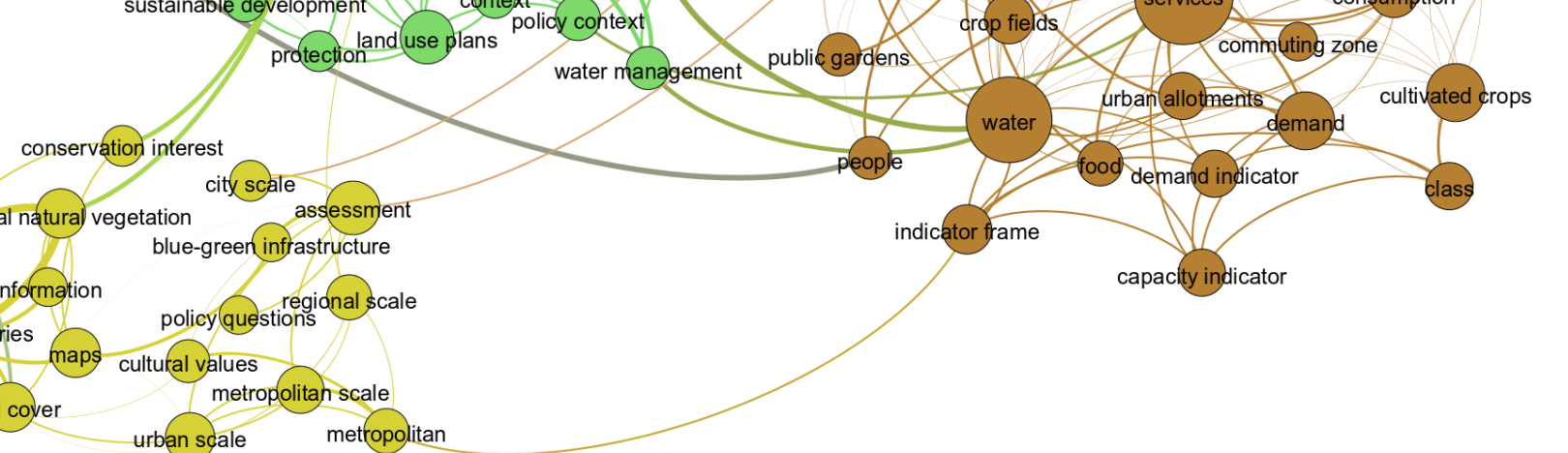

(1) Economic valuation
(2) Types of NBS
(3) Ecosystem services provided by NBS
(4) NBS terms
(5) Economic values of nature

\section{Number of nodes}
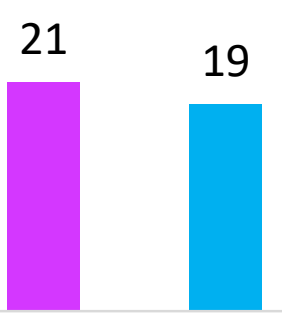

21

1

2

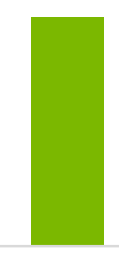

12

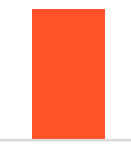

11

3

4

5

\section{Accumulated Degree}

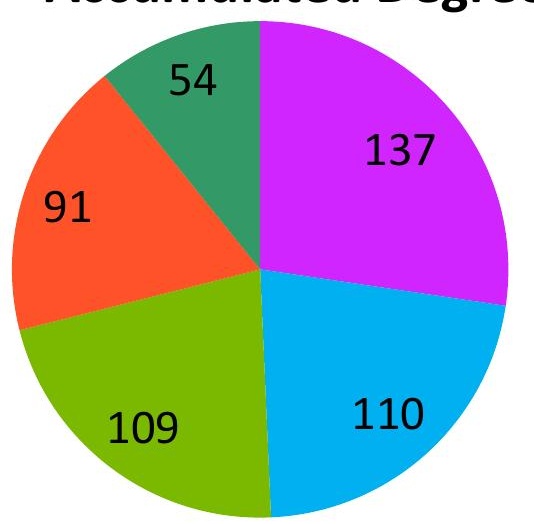

Node Degree

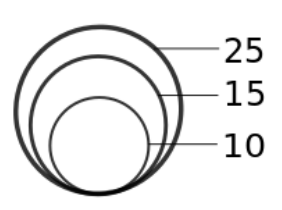

particular study monetisation literature differences urban nature un functions

evidence base selection nature

selection location nature monetary values
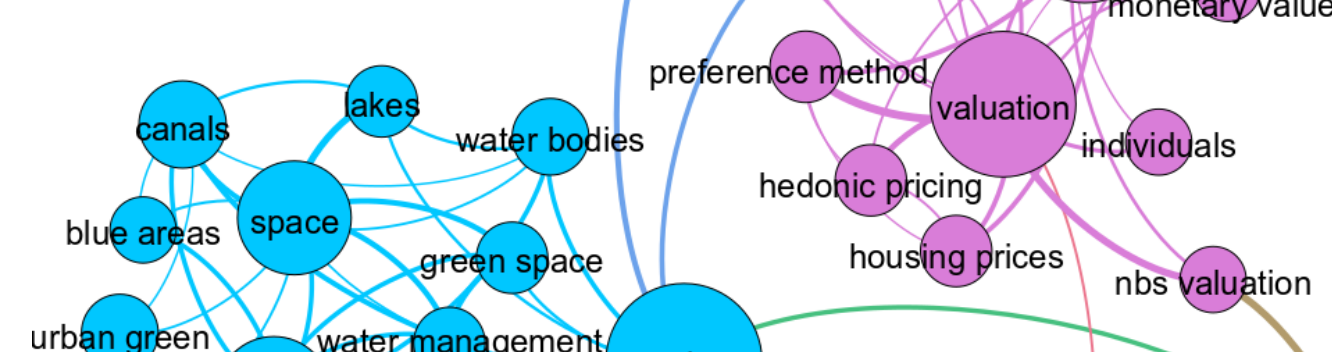

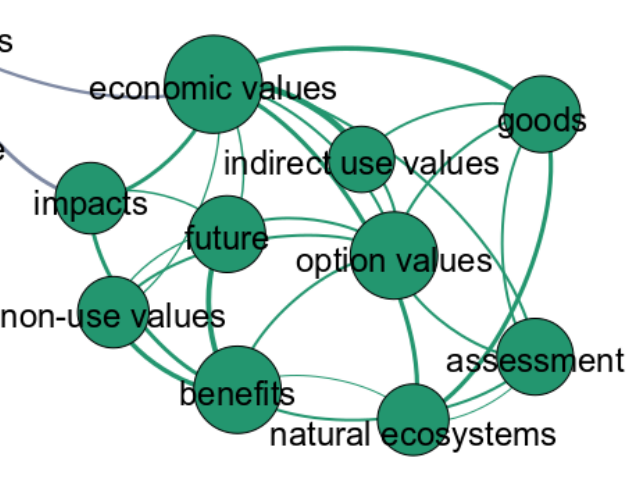




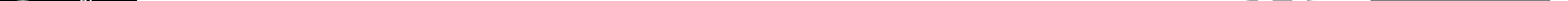

